\title{
Variabilidade fenotípica na síndrome do cromossomo supernumerário der(22)t(11;22) (síndrome de Emanuel)
}

\author{
Phenotypical variability in supernumerary chromosome der(22)t(11;22) syndrome (Emanuel syndrome)
}

\author{
Rafael Fabiano M. Rosa ${ }^{1}$, Juliana Nunes Pfeil2, Paulo Ricardo G. Zen³, Rosana Cardoso M. Rosa ${ }^{4}$, Carla Graziadio ${ }^{5}$, \\ Giorgio Adriano Paskulin 6
}

\section{RESUMO}

Objetivo: Relatar dois pacientes com a síndrome de Emanuel (SE) ou cromossomo supernumerário der(22) $\mathrm{t}(11 ; 22)$, secundária a translocações balanceadas familiares, apresentando fenótipos distintos.

Descrição dos casos: O primeiro paciente é uma menina branca de cinco anos de idade, apresentando hipotonia, atraso no desenvolvimento neuropsicomotor, movimentos estereotipados, microcefalia, ptose palpebral, orelhas proeminentes, fossetas e apêndices pré-auriculares, e imperfuração anal. As avaliações adicionais identificaram hipoplasia cerebral e estenose da válvula pulmonar. Possuía história também de laringotraqueomalácia e fenda palatina. O segundo paciente é um menino branco de seis meses de idade com hipotonia, movimentos coreoatetóticos, déficit de crescimento, microcefalia, microssomia hemifacial, fenda palatina, microtia, apêndices pré-auriculares e polegares proximalmente implantados. A ecocardiografia demonstrou estenose da válvula pulmonar, comunicação interatrial e interventricular, persistência do canal arterial e da veia cava superior esquerda. A radiografia de tórax identificou uma costela cervical. O cariótipo por bandas GTG mostrou a presença, em ambos os pacientes, de um cromossomo adicional $\operatorname{der}(22) \mathrm{t}(11 ; 22)$, secundário a uma translocação balanceada materna no primeiro caso e paterna no segundo caso.
Comentários: Apesar de a primeira paciente apresentar achados frequentes da SE, o caso adicional representa a segunda descrição da literatura com um fenótipo de espectro óculo-aurículo-vertebral (EOAV). Assim, ambos salientam a variabilidade clínica observada na SE e a importância da avaliação cariotípica em indivíduos com fenótipo de EOAV.

Palavras-chave: translocação genética; aberrações cromossômicas; fenótipo; síndrome de Goldenhar; aconselhamento genético.

\section{ABSTRACT}

Objective: To report two patients with Emanuel syndrome (ES) or supernumerary chromosome der(22)t(11;22), secondary to familial balanced translocations, presenting distinct phenotypes.

Cases description: The first patient was a five-year-old white girl presenting hypotonia, neuropsychomotor delay, stereotypic movements, microcephaly, ptosis, prominent ears, preauricular pits with skin tag, and imperforate anus. Additional evaluations identified cerebral hypoplasia and pulmonar valvar stenosis. She also had laryngotracheomalacia and cleft palate. The second patient was a six months white boy with hypotonia, choreoatetotic movements, growth retardation, microcephaly, hemifacial microsomia,
Instituição: Universidade Federal de Ciências da Saúde de Porto Alegre (UFCSPA) e Complexo Hospitalar Santa Casa de Porto Alegre (CHSCPA), Porto Alegre, RS, Brasil

'Doutorando pelo Programa de Pós-Graduação em Patologia da UFCSPA; Geneticista Clínico da UFCSPA e do CHSCPA, Porto Alegre, RS, Brasil

${ }^{2}$ Aluna do Curso de Medicina da UFCSPA, Porto Alegre, RS, Brasil

${ }^{3}$ Doutor pelo Programa de Pós-Graduação em Patologia da UFCSPA; Professor Adjunto da Disciplina de Genética Clínica e do Programa de Pós-Graduação em Patologia da UFCSPA, Porto Alegre, RS, Brasil

${ }^{4}$ Médica Residente do Programa de Residência Médica em Pediatria do Grupo Hospitalar Conceição, Porto Alegre, RS, Brasil

${ }^{5}$ Mestre pelo Programa de Pós-Graduação em Genética e Biologia Molecular da Universidade Federal do Rio Grande do Sul (UFRGS); Professora Assistente da Disciplina de Genética Clínica da UFCSPA, Porto Alegre, RS, Brasil
${ }^{6}$ Doutor pelo Programa de Pós-Graduação em Genética e Biologia Molecular da UFRGS; Professor Associado da Disciplina de Genética Clínica e do Programa de Pós-Graduação em Patologia da UFCSPA, Porto Alegre, RS, Brasil

Endereço para correspondência:

Giorgio Adriano Paskulin

Rua Sarmento Leite, 245, sala 403 - Centro

CEP 90050-170 - Porto Alegre/RS

E-mail: paskulin@ufcspa.edu.br

Conflito de interesse: nada a declarar

Recebido em:22/7/2009

Aprovado em:17/12/2009 
cleft palate, microtia, preauricular skin tags and thumbs proximally placed. Echocardiography disclosed pulmonary valvar stenosis, interatrial and ventricular septal defects, patent ductus arteriosus and persistence of left upper vein cava. Radiography study of thorax identified a cervical rib. GTG-Banding karyotype showed, in both patients, a supernumerary chromosome der(22)t $(11 ; 22)$ secondary in the first case to a maternal balanced translocation and in the second one to a paternal translocation.

Comments: The first patient presented frequent features of ES, but the second one is the second description in the literature of a phenotype of oculo-auriculo-vertebral spectrum (OAVS). Thus, both patients highlight the clinical variability observed in ES and the importance of the karyotype analysis in patients with OAVS phenotype.

Key-words: translocation, genetic ; chromosome aberrations; phenotype; Goldenhar syndrome; genetic counseling.

\section{Introdução}

A síndrome de Emanuel (OMIM 609029)(1), também conhecida como síndrome do cromossomo supernumerário $\operatorname{der}(22) t(11 ; 22)$ ou da trissomia parcial do cromossomo 22, é uma doença genética rara causada pela presença de um cromossomo marcador adicional secundário a uma translocação entre os cromossomos 11 e 22. Esse rearranjo cromossômico é a translocação recíproca mais comum em humanos. Atualmente, sabe-se que as regiões 11q23 e 22q11 apresentam repetições palindrômicas ricas em repetições AT (palindromic AT-rich repeats - PATTRs) que predispõem à formação dessa translocação ${ }^{(2-4)}$. A síndrome é clinicamente caracterizada por importante deficiência cognitiva, anomalias craniofaciais, malformações cardíacas e anormalidades renais ${ }^{(5-9)}$. Entretanto, considerável variabilidade no fenótipo tem sido observada na literatura ${ }^{(2,7,9)}$.

O espectro óculo-aurículo-vertebral (OMIM 164210) $)^{(1)}$, chamado de microssomia hemifacial ou síndrome de Goldenhar, é considerado uma condição heterogênea com fenótipo e etiologia variáveis. Apesar do fato de a maior parte dos casos não apresentar uma causa conhecida, diferentes anormalidades cromossômicas, epigenéticas e gênicas, bem como exposições a teratógenos têm sido descritas em associação a esse fenótipo. Sua prevalência é estimada entre um para 5.600 a 45 mil nascidos vivos. É clinicamente caracterizado pelo envolvimento assimétrico de estruturas originárias dos primeiros arcos branquiais. Entretanto, achados em outros órgãos ou sistemas, como cérebro, coração, membros e rins, são frequentemente observados ${ }^{(10-14)}$.

O objetivo deste artigo foi relatar dois novos pacientes com a síndrome do cromossomo supernumerário $\operatorname{der}(22) \mathrm{t}(11 ; 22)$, secundária a translocações balanceadas parentais, um com achados típicos da síndrome de Emanuel e outro com um fenótipo de espectro óculo-aurículo-vertebral.

\section{Descrição dos casos}

\section{Caso 1}

Menina caucasiana de dois anos e quatro meses de idade, segunda filha de pais jovens, hígidos e não-consanguíneos. A história familiar era negativa para doenças genéticas ou abortamentos. Ela nasceu após 34 semanas de uma gestação sem intercorrências por parto vaginal e apresentação cefálica, pesando $2600 \mathrm{~g}$ (percentil de 50 a 90), medindo $48 \mathrm{~cm}$ (percentil de 50 a 90), perímetro cefálico de $31 \mathrm{~cm}$ (percentil de 10 a 50) e Apgar de 8 e 9 no $1^{\circ}$ e $5^{\circ}$ minutos, respectivamente. A criança evoluiu com infecções respiratórias frequentes e sepse, necessitando de várias hospitalizações. A presença de laringotraqueomalácia foi posteriormente observada. A cirurgia de correção de palato fendido foi realizada aos dois anos.

Aos cinco anos de idade, seu peso era de $13400 \mathrm{~g}$ (< percentil 3), sua altura $106 \mathrm{~cm}$ (percentil 25 a 50 ) e seu perímetro cefálico, $47,5 \mathrm{~cm}$ ( $<$ percentil 2$)$. Ptose palpebral e pregas epicânticas bilaterais, filtro longo e profundo, micrognatia, orelhas proeminentes, fossetas pré-auriculares bilaterais e apêndice pré-auricular na orelha direita foram também observados (Figura 1). O ânus era imperfurado com uma fístula perineal e os pés eram planos. A avaliação neurológica identificou hipoatividade e importante hipotonia. A tomografia computadorizada (TC) de crânio mostrou ventrículos dilatados secundários à hipoplasia cerebral. A avaliação cardiológica verificou uma estenose leve de válvula pulmonar. O ultrassom abdominal foi normal (Tabela 1).

A criança apresentava importante atraso de desenvolvimento neuropsicomotor, com comportamento autista. Adquiriu sustento cefálico aos cinco meses de vida, sentou sem apoio aos quatro anos e começou a ficar de pé aos cinco anos. Nesta idade, ainda não caminhava ou falava e possuía dificuldade para segurar objetos. Ela apresentava irritabilidade, bem como movimentos estereotipados das mãos e interação com objetos não normalmente previstos para uma criança orientada (Tabela 1). 

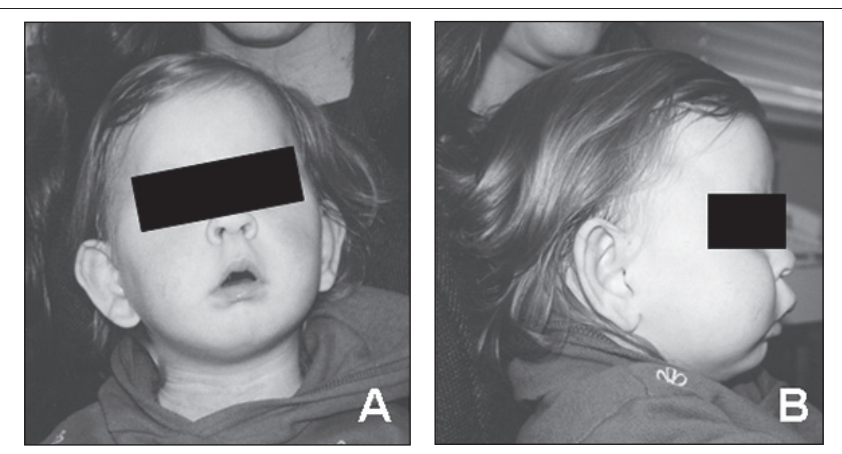

Figura 1 - Aparência craniofacial da paciente de um a 2 anos e quatro meses de idade ( $\mathrm{e}$ e $\mathrm{B}$ ). Notar especialmente o filtro longo, a micrognatia e as orelhas proeminentes.

A análise cromossômica através do cariótipo de alta resolução por bandas GTG ( $\geq 550$ bandas) mostrou a presença de um cromossomo marcador adicional. A análise cariotípica dos pais verificou que esse cromossomo marcador era secundário a uma translocação balanceada materna entre os cromossomos 11 e 22 (Figura 2). Assim, o cariótipo da paciente foi interpretado como 47, XX, +der(22)t(11;22)(q23;q11)mat.

\section{Caso 2}

Menino caucasiano de seis meses e 20 dias de vida, o segundo filho de pais jovens e não-consanguíneos. A história familiar era negativa para doenças genéticas ou abortamentos. A criança nasceu após 37 semanas de gestação por parto cesáreo, apresentação pélvica, pesando $3200 \mathrm{~g}$ (percentil 50 a 75) e medindo $49 \mathrm{~cm}$ (percentil 25 a 50). A mãe possuía história de asma. Houve um sangramento vaginal no primeiro mês de gravidez, perda de líquido amniótico e contrações durante toda a gestação. A mãe negou o uso de fumo, álcool ou outros teratógenos.

Aos seis meses de idade, a criança apresentava peso de $4365 \mathrm{~g}(<$ percentil 3), comprimento de 61,5cm (<percentil 3) e perímetro cefálico de $39,5 \mathrm{~cm}$ (<percentil 2). Mostrava região occipital proeminente, microssomia hemifacial à esquerda, ptose palpebral bilateral, narinas antevertidas e fenda palatina. As orelhas eram assimétricas com microtia e dois apêndices pré-auriculares à esquerda e microtia com presença de apenas o lobo e apêndices pré-auriculares entre a orelha e a boca à direita. $\mathrm{O}$ pescoço era longo, os polegares proximalmente implantados, havia hidrocele comunicante e fosseta sacral. A avaliação neurológica identificou hipotonia, movimentos coreoatetóticos, opistótono e sinais de envolvimento piramidal. A TC de crânio mostrou somente ventrículos levemente aumentados. A ecocardiografia detectou

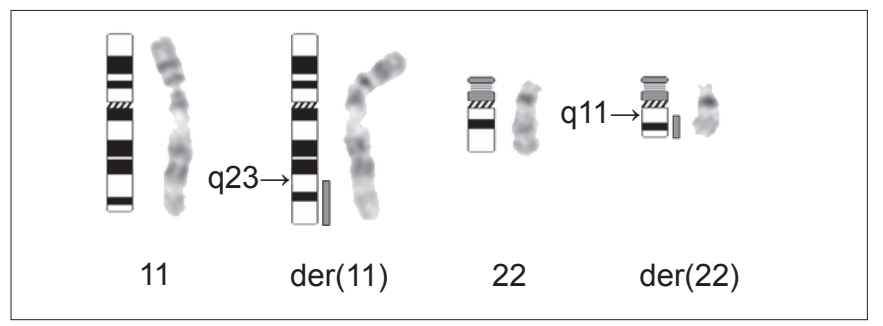

Figura 2 - Cariótipo parcial por banda GTG e ideograma mostrando a translocação recíproca e balanceada entre um cromossomo 11 [der(11)] e um cromossomo 22 [der(22)] apresentada pelos pais dos pacientes (as barras verticais em cinza indicam os segmentos cromossômicos translocados). Nos pacientes observou-se somente o adicional der(22).

um moderado defeito do septo atrial do tipo ostium secundum, defeito do septo ventricular do tipo perimembranoso, estenose leve da válvula pulmonar, ducto arterioso patente, persistência da veia cava superior esquerda e hipertensão pulmonar secundária. O estudo radiográfico do tórax e da coluna mostrou uma costela cervical à direita. $\mathrm{O}$ ultrassom abdominal e a função da tireoide foram normais (Tabela 1).

O cariótipo por bandas GTG ( $\geq 550$ bandas) identificou a presença de um cromossomo marcador adicional. Os estudos dos cromossomos dos pais mostraram que esse marcador era originário de uma translocação paterna entre os cromossomos 11 e 22 [47,XY, $+\operatorname{der}(22) \mathrm{t}(11 ; 22)(\mathrm{q} 23 ; \mathrm{q} 11)$ pat $]$ (Figura 2).

\section{Discussão}

Pacientes com a síndrome de Emanuel mostram trissomia parcial das regiões $11 \mathrm{q} 23 \rightarrow$ qter e 22 pter $\rightarrow \mathrm{q} 11$. Muitas das características clínicas apresentadas pelos pacientes são consideradas secundárias ao segmento trissômico do cromossomo 22. Embora todos os indivíduos contem com trissomia envolvendo regiões cromossômicas similares, eles não desenvolvem o mesmo fenótipo ${ }^{(2-4)}$. Relatos recentes têm tentado delinear o espectro fenotípico associado à síndrome. Achados craniofaciais são usualmente caracterizados por olhos profundos, hipotelorismo ocular, nariz achatado, lábio superior proeminente, fenda palatina e anomalias de orelha que incluem fossetas e apêndices pré-auriculares. Importante atraso de desenvolvimento neuropsicomotor e de fala, movimentos estereotipados com as mãos, hipotonia, defeitos cardíacos, anomalias genitais masculinas e anormalidades renais são também achados frequentes ${ }^{(5-9)}$.

Apesar de o nosso primeiro paciente mostrasse vários achados similares aos da maioria dos casos da síndrome de 
Tabela 1 - Comparação entre os achados clínicos apresentados pelos dois pacientes com os descritos na literatura para síndrome de Emanuel e de espectro óculo-aurículo-vertebral

\begin{tabular}{|c|c|c|c|c|c|}
\hline & \multicolumn{4}{|c|}{ Síndrome de Emanuel } & \multirow{2}{*}{$\begin{array}{l}\text { Espectro óculo- } \\
\text { aurículo-vertebral }\end{array}$} \\
\hline & Paciente 1 & Paciente 2 & Balci et a/(11) & Literatura* & \\
\hline \multicolumn{6}{|l|}{ Crescimento } \\
\hline Déficit de crescimento & + & + & & ++ & + \\
\hline \multicolumn{6}{|l|}{ Neurológico } \\
\hline Hipoplasia cerebral & + & + & + & + & + \\
\hline Hipoplasia do corpo caloso & & & + & + & + \\
\hline RDNPM & + & NA & + & ++ & + \\
\hline Atraso de fala & + & NA & + & ++ & + \\
\hline Comportamento autista & + & NA & & + & + \\
\hline Movimentos atetóticos & & + & & + & \\
\hline Hipotonia & + & + & + & ++ & + \\
\hline \multicolumn{6}{|l|}{ Craniofacial } \\
\hline Microcefalia & + & + & + & ++ & + \\
\hline Microssomia hemifacial & & + & + & + & ++ \\
\hline Ptose palpebral & + & + & & + & + \\
\hline Microftalmia & & & + & + & + \\
\hline Pregas epicânticas & + & & & + & + \\
\hline Estrabismo & & & + & + & + \\
\hline Filtro longo & + & & & ++ & + \\
\hline Fenda palatina & + & + & + & ++ & + \\
\hline Micro/retrognatia & + & & + & ++ & ++ \\
\hline Orelhas grandes & & & + & + & \\
\hline Microtia & & + & & + & ++ \\
\hline $\begin{array}{l}\text { Fosseta ou apêndices pré- } \\
\text { auriculares }\end{array}$ & + & + & + & ++ & ++ \\
\hline Perda auditiva condutiva & NE & NE & + & + & ++ \\
\hline \multicolumn{6}{|l|}{ Pescoço/tórax } \\
\hline Pescoço longo & & + & & + & \\
\hline Laringotraqueomalácia & + & & & + & + \\
\hline Costela cervical & & + & & + & + \\
\hline $\begin{array}{l}\text { Vértebras cervicais em } \\
\text { bloco }\end{array}$ & & & + & + & ++ \\
\hline $\begin{array}{l}\text { Defeitos cardíacos } \\
\text { congênitos }\end{array}$ & + & + & & ++ & ++ \\
\hline \multicolumn{6}{|l|}{ Membros superiores } \\
\hline Anomalias radiais & & + & & + & + \\
\hline \multicolumn{6}{|l|}{ Abdome } \\
\hline Anormalidades renais & & & + & + & + \\
\hline Hérnia umbilical/inguinal & & & + & + & + \\
\hline \multicolumn{6}{|l|}{ Anogenital } \\
\hline Ânus imperfurado & + & & & + & + \\
\hline Fosseta sacral & & + & & + & \\
\hline \multicolumn{6}{|l|}{ Membros inferiores } \\
\hline Pés planos & + & & + & & \\
\hline
\end{tabular}

RDNPM: retardo de desenvolvimento neuropsicomotor; NA: não se aplica; +: achados presentes; ++: achados mais frequentes; NE: não-avaliado;

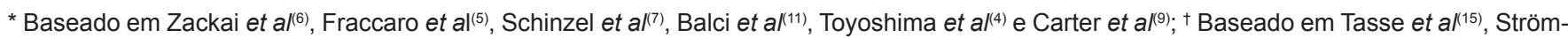
land et $a^{(14)}$ e Engiz et $a^{(16)}$. 
Emanuel descritos na literatura, o segundo possuía algumas manifestações não-usuais. Havia microssomia hemifacial e microtia assimétrica, em adição a fossetas e apêndices préauriculares, anomalia de costela e polegares proximalmente implantados, achados do espectro óculo-aurículo-vertebral $^{(1,14,15)}$. Essa é uma condição complexa e etiologicamente heterogênea, caracterizada por anormalidades dos arcos branquiais que envolvem especialmente a face, olhos, orelhas e vértebras. Alterações cromossômicas têm sido descritas em alguns pacientes com esse fenótipo ${ }^{(12,14,16)}$ e o presente relato representou o segundo caso com um cromossomo supernumerário $\operatorname{der}(22) \mathrm{t}(11 ; 22)^{(11,17)}$. Por outro lado, não se pode excluir a possibilidade de que os eventos vasculares ocorridos durante a gestação (sangramentos vaginais e contrações uterinas), fatores possivelmente associados à etiologia do espectro óculo-aurículo-vertebral ${ }^{(10,13)}$, possam ter tido algum efeito sobre o seu fenótipo.

\section{Referências bibliográficas}

1. Online Mendelian Inheritance in Man, OMIM (TM) [homepage on the Internet]. Baltimore e Bethesda: BeMcKusick-Nathans Institute for Genetic Medicine, Johns Hopkins University and National Center for Biotechnology Information, National Library of Medicine [cited 2009 Jul 15]. Available from: http://www. ncbi.nlm.nih.gov/omim/

2. Yosunkaya Fenerci E, Guven GS, Kuru D, Yilmaz S, Tarkan-Argüden Y, Cirakoglu A et al. Supernumerary chromosome der(22)t(11;22): Emanuel syndrome associates with novel features. Genet Couns 2007;18:401-8.

3. Medne L, Zackai EH, Emanuel BS [homepage on the Internet]. Emanuel syndrome. Gene Reviews [cited 2009 Jul 15]. Available from: www.ncbi.nem. nih.gov/bookshelf/br.fcgi?book=gene\&pait=emanuel

4. Toyoshima M, Yonee C, Maegaki Y, Yamamoto T, Shimojima K, Maruyama S et al. Vertebral fusion in a patient with supernumerary-der(22)t(11;22) syndrome. Am J Med Genet A 2009;149A:1722-6.

5. Fraccaro M, Lindsten J, Ford CE, Iselius L. The 11q;22q translocation: a European collaborative analysis of 43 cases. Hum Genet 1980;56:21-51.

6. Zackai EH, Emanuel BS. Site-specific reciprocal translocation, $t(11 ; 22)$ (q23;q11), in several unrelated families with 3:1 meiotic disjunction. Am J Med Genet 1980;7:507-21.

7. Schinzel A, Schmid W, Auf der Maur P, Moser H, Degenhardt KH, Geisler M et al. Incomplete trisomy 22. I. Familial 11/22 translocation with $3: 1$ meiotic disjunction. Delineation of a common clinical picture and report of nine new cases from six families. Hum Genet 1981;56:249-62.

8. Gremeau AS, Coste K, Blanc P, Goumy C, Francannet C, Dechelotte PJ et al. Congenital diaphragmatic hernia and genital anomalies: Emanuel syndrome. Prenat Diagn 2009;29:816-8.

9. Carter MT, St Pierre SA, Zackai EH, Emanuel BS, Boycott KM. Phenotypic delineation of Emanuel syndrome (supernumerary derivate 22 syndrome):
Na maioria dos indivíduos com a síndrome de Emanuel, como ocorreu com nossos pacientes, um dos pais apresenta uma translocação balanceada $\mathrm{t}(11 ; 22)(\mathrm{q} 23 ; \mathrm{q} 11.2)$ e é fenotipicamente normal. Seu risco para gerar um recém-nascido com o der(22) supernumerário varia de 1,8 a 5,6\% e, para abortamento espontâneo, de 23 a 37\%. Indivíduos com a síndrome de Emanuel são considerados improváveis de se reproduzir devido à sua importante deficiência cognitivaa ${ }^{(3,5,6,17)}$.

Nossos relatos salientam a variabilidade clínica observada na síndrome de Emanuel. Em particular, o segundo caso enfatiza a possível sobreposição com o fenótipo verificado no espectro óculo-aurículo-vertebral. Por outro lado, como bem colocado por Balci et al $l^{(11)}$, destaca-se o papel da análise cariotípica em pacientes com espectro óculo-aurículovertebral. Isso é importante para determinar a etiologia e o correto aconselhamento genético a ser ministrado ao paciente e à sua família.
Clinical features of 63 individuals. Am J Med Genet A 2009;149A: 1712-21.

10. Cohen MM Jr, Rollnick BR, Kaye Cl. Oculoauriculovertebral spectrum: an updated critique. Cleft Palate J 1989;26:276-86.

11. Balci S, Engiz O, Yilmaz Z, Baltaci V. Partial trisomy $(11 ; 22)$ syndrome with manifestations of Goldenhar sequence due to maternal balanced $t(11 ; 22)$. Genet Couns 2006;17:281-9.

12. Castori M, Brancati F, Rinaldi R, Adami L, Mingarelli R, Grammatico P et al. Antenatal presentation of the oculo-auriculo-vertebral spectrum (OAVS). Am J Med Genet A 2006;140:1573-9.

13. Wieczorek D, Ludwig M, Boehringer S, Jongbloet PH, Gillessen-Kaesbach G, Horsthemke B. Reproduction abnormalities and twin pregnancies in parents of sporadic patients with oculo-auriculo-vertebral spectrum/Goldenhar syndrome. Hum Genet 2007;121:369-76.

14. Strömland K, Miller M, Sjögreen L, Johansson M, Joelsson BM, Billstedt E et al. Oculo-auriculo-vertebral spectrum: associated anomalies, functional deficits and possible developmental risk factors. Am J Med Genet A 2007;143A: 1317-25.

15. Tasse C, Böhringer S, Fischer S, Lüdecke HJ, Albrecht B, Horn D et al. Oculo-auriculo-vertebral spectrum (OAVS): clinical evaluation and severity scoring of 53 patients and proposal for a new classification. Eur J Med Genet 2005;48:397-411.

16. Engiz O, Balci S, Unsal M, Ozer S, Oguz KK, Aktas D. 31 cases with oculoauriculovertebral dysplasia (Goldenhar syndrome): clinical, neuroradiologic, audiologic and cytogenetic findings. Genet Couns 2007;18:277-88.

17. Iselius L, Lindsten J, Aurias A, Fraccaro M, Bastard C, Bottelli AM et al. The 11q;22q translocation: a collaborative study of 20 new cases and analysis of 110 families. Hum Genet 1983;64:343-55. 